\title{
Uma Plataforma Ubíqua e Pervasiva para Distribuição de Conteúdo em Ambientes Educacionais
}

\author{
André Felipe Monteiro ${ }^{1}$, Dalbert Matos Mascarenhas ${ }^{1}$, Laura Silva de Assis ${ }^{1}$ \\ ${ }^{1}$ Centro Federal de Educação Tecnológica Celso Suckow da Fonseca (CEFET/RJ) \\ Rua do Imperador, 971 - Centro - Petrópolis - RJ - Brasil
}

\begin{abstract}
In most educational environments, the process of communication with students is performed on ad hoc basis, with low standardization and a little interaction of side participation. However, a more dynamic communication is demanded by the young profile of most students. In this way, mobile devices are presented as an efficient and flexible channel to reach this public. In this scenario, we present a ubiquitous and pervasive platform to provide the distribution of educational content using a sensitive model context. Thus, the platform can satisfy the specific demands informed by the user, and also respond to the current context, sending personalized content according to predefined aspects such as location, profile, among others.
\end{abstract}

Resumo. Nos dias atuais, em grande parte dos ambientes educacionais, o processo de comunicação com os alunos é realizada de forma ad hoc, com baixa padronização e pouca interação entre as partes. Entretanto, uma comunicação mais dinâmica é demandada pelo perfil jovem da maior parte dos alunos. Desta forma, os dispositivos móveis apresentam-se como um canal eficiente e flexível de atingir esse público. Nesse cenário, apresentamos uma plataforma ubíqua e pervasiva para prover a distribuição de conteúdo educacional utilizando um modelo sensivel a contexto. Assim, a plataforma pode atender às demandas específicas informadas pelo usuário, e também reagir ao contexto atual do mesmo, enviando conteúdos personalizados conforme aspectos pré-definidos como localização, perfil, dentre outros.

\section{Introdução}

O processo de comunicação entre o público interno em ambientes educacionais (professores, técnicos administrativos, alunos, colaboradores, etc.) é realizado em geral de forma ad hoc, onde múltiplas plataformas de comunicação são utilizadas de acordo com a conveniência dos envolvidos. Desta forma, podemos observar o uso de e-mails, página pessoal de professores e instituições, grupos fechados em redes sociais, dentre outras alternativas, para implementar um canal de comunicação em ambientes educacionais.

Entretanto, com o avanço tecnológico e as múltiplas plataformas de informação disponíveis, o processo de comunicação principalmente entre professores e alunos necessita ser dinâmico e eficiente, de forma a complementar o conteúdo apresentado e discutido em sala de aula. Sob a perspectiva dos alunos, há um ambiente heterogêneo de comunicação, visto que cada professor pode optar por um canal de comunicação diferente. Este cenário pode acarretar em eventuais falhas de comunicação e desinteresse dos alunos em obter conteúdo educacional, como material didático complementar, listas de exercício, etc., conforme analisado em [Silva et al. 2011]. 
Para contornar este gargalo de comunicação em ambientes educacionais, apresentamos uma plataforma ubíqua e pervasiva para distribuição de conteúdo utilizando a rede de dados sem fio da instituição. Os alunos receberão conteúdo de seus professores, ou até mesmo comunicações institucionais de departamentos e setores, como biblioteca, laboratórios, etc., por meio de dispositivos móveis (smart phones e tablets) utilizando um modelo sensível a contexto. Portanto, a plataforma pode atender ao interesse em conteúdos específicos previamente informados pelo usuário, e também reagir ao contexto atual do mesmo, enviando conteúdos personalizados de acordo com diversos aspectos como localização, perfil de acesso, dentre outros.

Além de prover uma solução eficiente para comunicação interna, esta plataforma também se mostra um ambiente multidisciplinar, com aplicação de diversas áreas da Computação como Redes, Programação, Otimização, dentre outras, que necessitam se relacionar de forma integrada e consistente. Ou seja, um ambiente ideal para aplicação prática de conhecimento dos alunos do curso de Engenharia de Computação do CEFET/RJ Campus Petrópolis, que participam do projeto como usuários finais da plataforma, ou em conjunto com os autores deste trabalho na modelagem e implementação da plataforma, por meio de bolsas de projetos PIBIC e de Extensão.

Optamos por utilizar dispositivos móveis como único canal de comunicação do usuário final com a plataforma em virtude da melhor usabilidade e da maior familiaridade dos jovens com esta tecnologia, conforme apontado em [Charland and Leroux 2011]. Além disso, uma avaliação da nossa plataforma junto ao público alvo do projeto é apresentada na Seção 5. Os resultados indicam que há um grande interesse dos alunos em participar da especificação e implementação da plataforma, ratificando a característica interdisciplinar e integradora da mesma.

Este trabalho está organizado da seguinte forma: na Seção 2 apresentamos os trabalhos relacionados, comparando a abordagem utilizada e as funcionalidades da plataforma junto à literatura. Na Seção 3 analisamos a rede em malha sem fio utilizada como ambiente de comunicação da plataforma. Já na Seção 4, discutimos os detalhes e a implementação da aplicação ubíqua e pervasiva projetada para uso dos alunos em seus dispositivos móveis. Na Seção 5 apresentamos os resultados de uma pesquisa de interesse realizada com o público alvo da plataforma, e finalmente na Seção 6 descrevemos as conclusões e os apontamentos para trabalhos futuros.

\section{Trabalhos relacionados}

Diversos trabalhos presentes na literatura abordam a implementação de uma plataforma para compartilhamento de conteúdo educacional. Em [Orlandi and Isotani 2012] é proposto um sistema para correção online de listas de exercício enviadas pelos professores de disciplinas através dos dispositivos móveis de alunos. Já em [Rabello et al. 2012] é apresentada uma plataforma para colaboração entre alunos, que trocam informações e experiências sobre as atividades designadas pelos professores.

Nossa solução possui similaridades com as ferramentas mencionadas, como a utilização de dispositivos móveis como canal de comunicação com os alunos, e o envio de material educacional através da plataforma. Entretanto, nosso trabalho se diferencia pela segmentação e personalização do conteúdo a ser enviado para cada aluno de acordo com o contexto associado ao mesmo. Desta forma, priorizamos o envio de um conteúdo 
mais seletivo e representativo sob o ponto de vista do usuário, evitando um processo de saturação de conteúdo e informações que poderia prejudicar a usabilidade dos usuários da plataforma.

Sob o ponto de vista do ambiente de aprendizado multidisciplinar viabilizado pelo processo de concepção e implementação da nossa plataforma, há trabalhos relevantes na literatura que analisam e propõe ambientes de aprendizagem e de troca de conteúdo educacional. Em geral, as disciplinas relacionadas à área de Programação são as mais frequentes na utilização de ambientes complementares para auxiliar o processo de aprendizagem. Em [Campos et al. 2015] e [Vahldick et al. 2015] um ambiente de jogos é utilizado para complementar o conteúdo destinado a disciplinas introdutórias de Programação. Já os trabalhos presentes em [Chaves et al. 2013] e [Santos et al. 2013] não abordam o aspecto da gamificação, mas também apresentam ambientes para apoiar o conteúdo de disciplinas de Programação.

Conforme pode ser observado, esses trabalhos não abrangem um escopo multidisciplinar, já que são destinados prioritariamente a disciplinas específicas da Computação. Por outro lado, nossa plataforma é abrangente no aspecto multidisciplinar, já que a mesma aborda de forma integrada várias áreas da Computação durante todo o processo de modelagem e implementação, conforme já discutido na Seção 1. Desta forma, podemos observar que a nossa plataforma é capaz de prover conteúdo e informações em ambientes educacionais baseados no contexto de cada usuário, além de se consolidar como um ambiente multidisciplinar para atuação de alunos e professores.

\section{A rede de comunicação proposta}

A utilização de redes em malha sem fio (wireless mesh networks) para prover acesso à internet em locais públicos ou privados mostra-se uma solução eficiente quando há necessidade de cobertura de uma área geograficamente grande. Isso ocorre em virtude da facilidade de implantação das redes em malha em áreas onde o uso de infraestrutura cabeada seria inviável.

Atualmente, muitas universidades utilizam as redes em malha sem fio para interligar prédios de seus campi e prover acesso à internet para seus alunos e colaboradores, além de a utilizarem como uma poderosa plataforma de teste e pesquisa. Algumas cidades também começam a utilizar esta solução para prover conexão entre pontos relevantes de seu território, como prédios públicos, museus, praças, viabilizando o acesso à internet para a comunidade em diversos pontos da cidade e a criação de cidades digitais, contribuindo assim para o avanço do processo de inclusão digital [Ahuja et al. 1993].

Uma das principais características das redes em malha está no uso de roteadores sem fio, geralmente instalados em locais fixos e com conexão à rede elétrica, que possuem um maior poder de processamento em relação aos roteadores móveis, que em geral apresentam suprimento de energia limitado. Uma arquitetura de uma rede em malha sem fio pode ser observada na Figura 1, onde cada nó representa um roteador e consequentemente um ponto de acesso distinto na rede.

Os roteadores presentes na rede em malha têm duas funções básicas: (i) encaminhar tráfego para os outros roteadores que fazem parte da rota de destino e (ii) receber e encaminhar tráfego para os nós clientes (dispositivos móveis de usuários). A auto- 


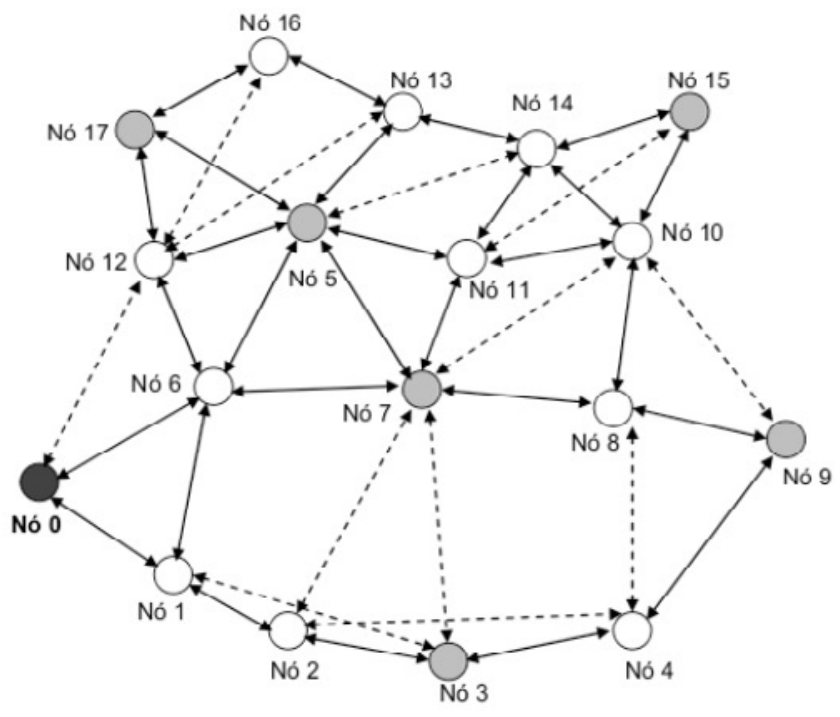

Figura 1. Arquitetura de uma rede em malha sem fio

organização e a auto-configuração devem ser características presentes nas redes em malha, de forma que a manutenção das conexões entre os roteadores seja automática. Tais características facilitam a inclusão de novos nós roteadores na rede, ampliando sua cobertura, além de as tornarem redes gerenciáveis e tolerantes a falhas, devido aos múltiplos caminhos possíveis criados entre nós roteadores e nós clientes[Mascarenhas et al. 2008].

Uma das vantagens de se utilizar as redes em malha é que elas oferecem um menor custo de infra-estrutura, dado que requerem menor número de pontos de acesso em comparação às redes cabeadas, proporcionando assim conectividade a uma área muito maior que as redes sem fio tradicionais. As redes em malha, ao contrário das redes ad-hoc nas quais se baseiam, não enfrentam restrições referentes à mobilidade e ao consumo de energia, pois normalmente os nós têm localização fixa e podem ser facilmente alimentados, tornando-os capazes de prover maior largura de banda e confiabilidade.

Uma infraestrutura de uma rede em malha sem fio permite que aplicações destinadas a dispositivos móveis utilizem a localização do ponto de acesso (roteador) para prover conteúdo específico destinado àquele local. Um possível exemplo é um usuário utilizando seu dispositivo móvel (smart phone, tablet, notebook) e conectado à rede em malha a partir do roteador $A$ que está instalado ao lado da biblioteca do campus. Este usuário pode receber em seu dispositivo informações relacionadas à biblioteca, como horário de funcionamento, espaços ainda disponíveis para estudo, quantidade de computadores livres para uso, etc. Da mesma forma, caso este mesmo usuário se desloque para o prédio onde está situado o laboratório de redes, que é atendido na rede em malha pelo roteador $B$, as informações enviadas serão outras, como horário de aulas, recados, quantidade de máquinas disponíveis, etc.

Conhecendo o local de onde o usuário faz o acesso na rede em malha sem fio, é possível personalizar o comportamento de uma aplicação para que ela se adeque à localização do usuário. Este conceito é utilizado em larga escala para aplicações ubíquas e pervasivas. De uma forma geral, este tipo de aplicação utiliza o paradigma cliente- 
servidor em sua arquitetura. Assim, o cliente é representado pelos dispositivos móveis dos usuários que enviarão requisições para os servidores de conteúdo, conforme indicado na Figura 2. Dado a possibilidade de identificar o local de origem da requisição, utilizando a identificação do roteador de acesso, o servidor que atender à requisição poderá enviar um conteúdo específico para o cliente.

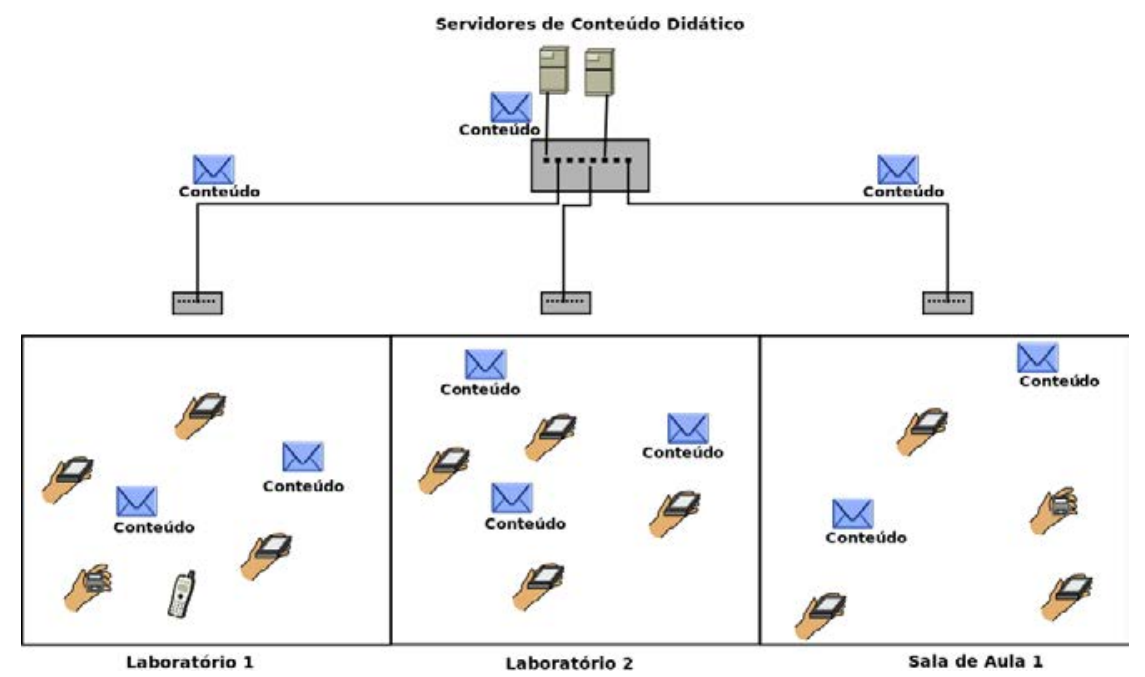

Figura 2. Modulo da arquitetura da rede

O projeto visa também restringir o impacto causado pela redundância de informações armazenadas e consequentemente trafegadas na rede de computadores. A Figura 3 exemplifica parte do escopo de armazenamento e distribuição do projeto. Para isto foram criadas estratégias de contenção para os recursos didáticos em redundância. Uma vez que os detalhes da rede de dados estão apresentados, descrevemos a seguir a aplicação cliente-servidor que irá distribuir os conteúdos educacionais aos usuários da plataforma.

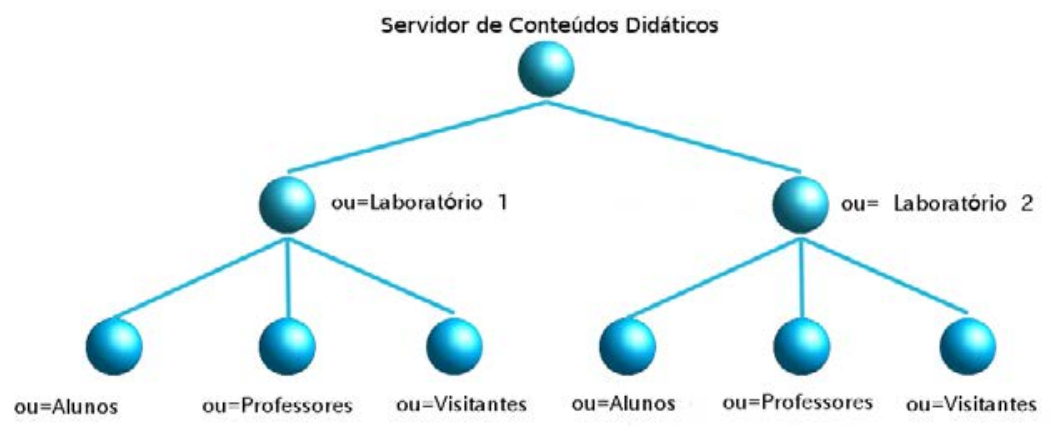

Figura 3. Escopo de Segmentação dos Conteúdos

\section{A plataforma de conteúdo}

A plataforma de conteúdo proposta utiliza uma arquitetura cliente-servidor, e se baseia em 3 componentes básicos. O primeiro consiste em uma Interface HTML onde é inserido 
o conteúdo que será distribuído aos usuários. Posteriormente, os conteúdos inseridos em formato texto, (recados, exercícios, etc.) e seus meta-dados de controle são convertidos em um arquivo Json antes do envio para o servidor. Caso o conteúdo inserido esteja na forma de arquivos de imagem (figuras, arquivos PDF, etc.), os mesmos são enviados para o servidor sem necessidade de conversão.

Um servidor Apa che é o segundo componente do sistema, sendo responsável por armazenar em um banco de dados SQL o conteúdo enviado pela Interface HTML, e disponibilizá-lo ao App. O terceiro componente é o App utilizado pelo aluno em seu dispositivo móvel para obter atualização de conteúdo através da rede em malha sem fio. Para esta ação, o App envia requisições HTTP ao servidor, que identifica a origem da requisição por meio do endereço IP dos pacotes recebidos. Essa identificação é verificada no servidor por meio de funções implementadas em PHP que manipulam uma tabela hash onde os endereços IP dos dispositivos móveis estão associados aos roteadores presentes na rede em malha. Ou seja, com base nos endereços IP das requisições podemos identificar o local onde o dispositivo móvel está presente e segmentar o conteúdo a ser enviado em resposta àquela requisição.

Utilizamos arquivos Json para realizar a comunicação entre os componentes do sistema em virtude de sua portabilidade entre plataformas, e pelo seu tamanho reduzido em relação a outras alternativas similares como o XML, por exemplo. Os trabalhos apresentados em [Nurseitov et al. 2009] e [Wang 2011] ratificam que o Json é mais eficiente que outras abordagens para a comunicação entre componentes cliente-servidor quando as plataformas Java e PHP são utilizadas. Em virtude de sua menor complexidade e tamanho, o arquivo J s on requer menos processamento do cliente Java, provendo inclusive um menor consumo de energia do dispositivo móvel, que é um dos principais gargalos dos smart phones e tablets atuais. A Figura 4 exemplica um arquivo Json.

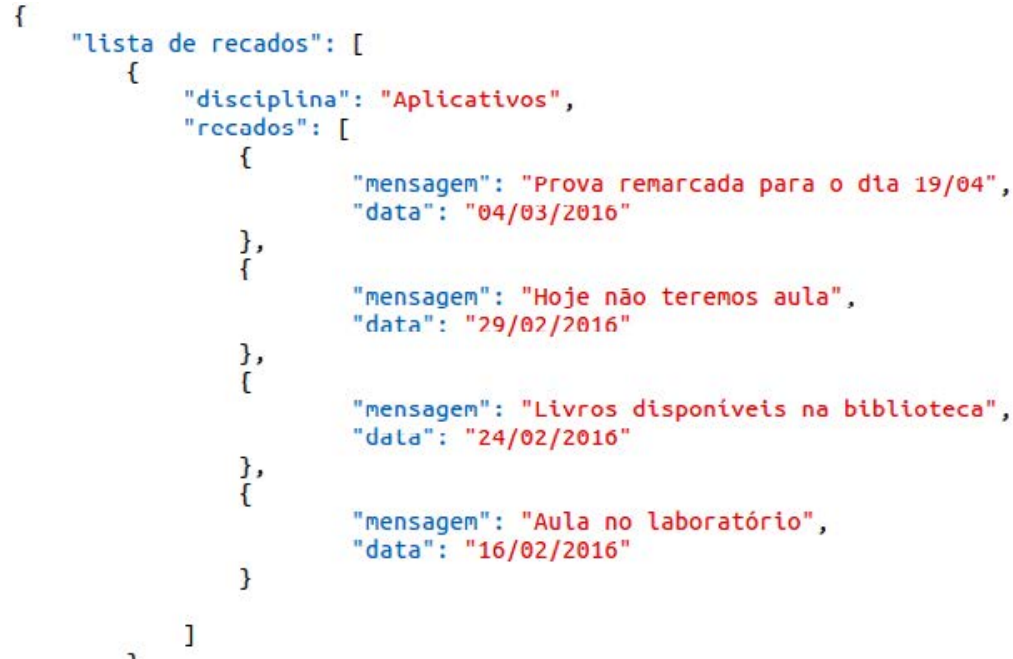

Figura 4. Arquivo Json confeccionado pelo servidor de conteúdo

A título de exemplo, o arquivo Json apresentado na Figura 4 foi confeccionado pelo servidor de conteúdo com os eventos de recados de uma disciplina fictícia de "Aplicativos" e enviado para processamento no App, possuindo um tamanho de $476 \mathrm{By}-$ 
tes. Caso essas informações fossem enviadas pelo servidor em um arquivo imagem PDF para apresentação no App, esse arquivo teria o tamanho de 15,3 kB. Ou seja, o tamanho do arquivo Js on é aproximadamente 30 vezes menor que o arquivo PDF.

Para a aplicação cliente implementamos um App na plataforma Android. Optamos por implementar um App, em detrimento a um cliente web a ser executado em um browse do dispositivo móvel, em virtude da melhor usabilidade e da maior familiaridade dos jovens com esta plataforma, conforme apontado em [Charland and Leroux 2011]. Utilizamos a linguagem Java para construir o App, já que a mesma oferece um suporte mais eficiente à API do Android, de acordo com [Gavalas and Economou 2011].

Na Figura 5 são apresentadas algumas telas do App implementado para a plataforma Android. A Figura 5(a) ilustra um menu do App onde o usuário pode verificar os recados, listas de exercícios e o material didático associados à localização do usuário dentro da rede em malha sem fio. Já a Figura 5(b) mostra os recados cadastrados da disciplina "Aplicativos" apresentados em ordenação cronológica no App, considerando que o usuário encontra-se localizado na sala de aula destinada à referida disciplina. Assim, caso o usuário se deslocasse para uma outra sala de aula que está associada a outro roteador na rede em malha, o conteúdo provido estaria relacionado à disciplina desta nova sala de aula, e não mais com a disciplina "Aplicativos".

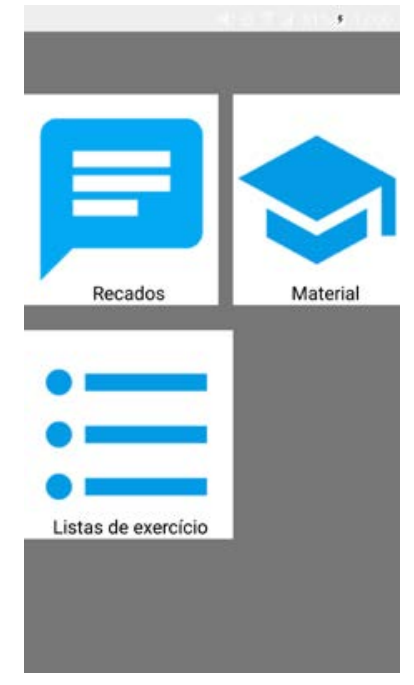

(a) Menu principal

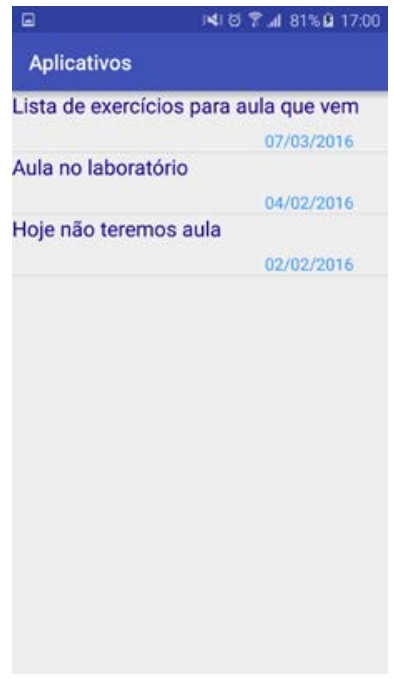

(b) Lista de recados

Figura 5. Exemplos de telas do App

\section{Receptividade da plataforma proposta}

Realizamos uma pesquisa com os alunos do curso de Engenharia de Computação a fim de obter a receptividade da plataforma proposta neste trabalho. Foi distribuído um questionário com três questões de múltipla escolha e tivemos um total de 58 questionários respondidos pelos alunos. O questionário fornecido contém as seguintes perguntas e suas respectivas alternativas de resposta:

1. Como você considera a comunicação entre o professor da disciplina e os alunos? Tal como envio de lista de exercícios, recados e avisos, material didático, etc. 
- (a) Ótimo

- (b) Bom

- (c) Regular

- (d) Ruim

- (e) Péssimo

- (f) Nenhuma das alternativas

2. Você considera que um aplicativo para dispositivos móveis onde fosse possível receber informações dos professores e da instituição de ensino por meio da rede sem fio interna do campus seria:

- (a) Imprescindível

- (b) Muito útil

- (c) Útil

- (d) Pouco útil

- (e) Sem utilidade

- (f) Nenhuma das alternativas

3. Como você gostaria de colaborar no projeto de construção desta plataforma?

- (a) No processo de desenvolvimento do App (dispositivo móvel e servidor)

- (b) No processo de configuração e instalação da rede em malha sem fio

- (c) No processo de teste, utilizando App como um usuário e provendo o feedback de suas experiências

- (d) Não tenho interesse em colaborar

Os resultados do questionário estão apresentados na Figura 6. A Figura 6(a) indica que há demanda para a plataforma proposta neste trabalho, visto que aproximadamente $42 \%$ dos alunos consideram a atual forma de comunicação professor-aluno como regular, ruim ou péssima. Além disso, a Figura 6(b) indica que aproximadamente $80 \%$ dos alunos entrevistados consideram que um App para prover conteúdo educacional complementar seria imprescindível, muito útil ou útil.

Por fim, apesar da implementação da plataforma ainda estar em caráter inicial, como apenas alguns protótipos funcionais em execução, os alunos se interessaram de forma significante pelo projeto. Conforme apresentado na Figura 6(c), em torno de $30 \%$ dos entrevistados manifestaram interesse em participar de atividades relacionadas à especificação e implementação do App e dos servidores de conteúdo. Já para participar de tarefas associadas à configuração e instalação da rede em malha sem fio, $15 \%$ dos entrevistados manifestaram interesse.

Em outras palavras, $45 \%$ do público entrevistado indicou o desejo de participar diretamente dos processos de modelagem, especificação, implementação e instalação da plataforma proposta neste trabalho. Esse relevante quantitativo ratifica o caráter catalisador e integrador do projeto, abrindo ótimos horizontes para atividades de pesquisa e extensão relacionadas a áreas abordadas pela plataforma. 


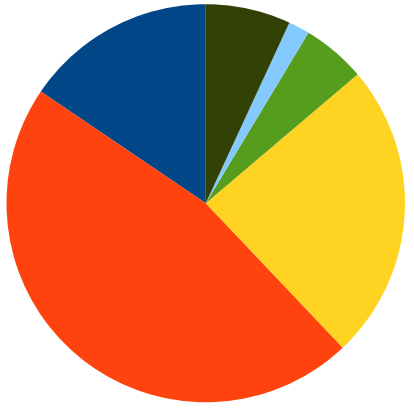

(a) Pergunta 1

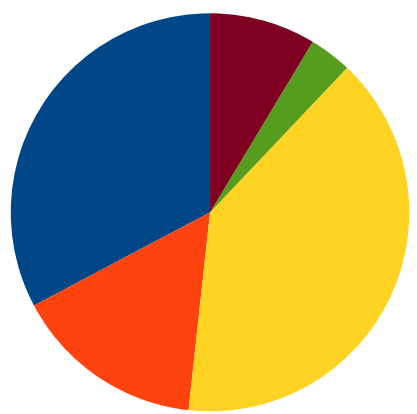

(c) Pergunta 3

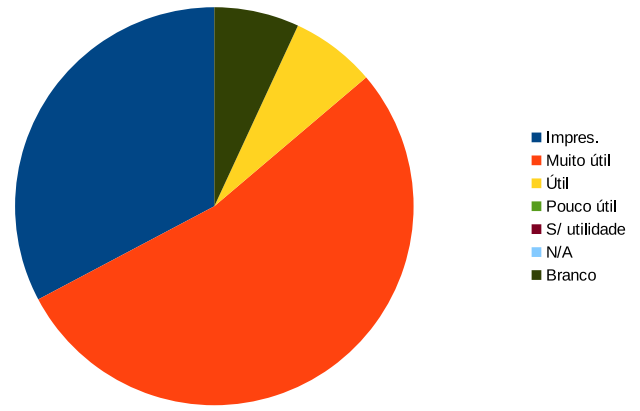

(b) Pergunta 2

\begin{tabular}{|c|c|c|c|}
\hline & Pergunta 1 & Pergunta 2 & Pergunta 3 \\
(a) & 9 & 19 & 19 \\
(b) & 27 & 31 & 09 \\
(c) & 14 & 04 & 23 \\
(d) & 03 & 0 & 02 \\
(e) & 0 & 0 & 0 \\
(f) & 1 & 0 & 0 \\
branco & 04 & 04 & 05 \\
\hline
\end{tabular}

(d) Resumo das respostas

Figura 6. Resultados dos questionários de avaliação

\section{Considerações finais}

Este trabalho apresentou uma plataforma ubíqua e pervasiva para distribuição de conteúdo em ambientes educacionais. As primeiras implementações mostraram a viabilidade da plataforma, ratificando seu aspecto multidisciplinar. Cabe ressaltar o potencial catalizador da solução proposta neste trabalho, atraindo interesse de alunos e professores para colaboração em todas as fases do projeto, com ótimas perspectivas para futuras atividades de pesquisa e extensão.

Dentre as próximas atividades previstas no projeto, pretendemos viabilizar um melhor escoamento dos dados trafegados na plataforma utilizando processos de otimização. Esses processos deverão otimizar o fluxo de dados na rede, evitando gargalos e sobrecargas em pontos específicos. Também desejamos implementar um cluster de servidores virtuais de conteúdo utilizando ferramentas de virtualização, alcançando uma maior flexibilidade no gerenciamento de recursos dos servidores, e viabilizando outras características relevantes como tolerância a falhas, economia de energia, etc. Por fim, agradecemos ao CNPq e ao CEFET/RJ pelas bolsas de iniciação científica e extensão, utilizadas pelos alunos de graduação em Engenharia de Computação que participaram deste projeto.

\section{Referências}

Ahuja, R. K., Magnanti, T. L., and Orlin, J. B. (1993). Network flows: theory, algorithms, and applications. Prentice Hall. 
Campos, A., Gardiman, R., and Madeira, C. (2015). Gamificação de uma disciplina introdutória de programação. $35^{\circ}$ Congresso da Sociedade Brasileira de Computação (CSBC'15), 23 Workshop sobre Educação em Computação (WEI' 15).

Charland, A. and Leroux, B. (2011). Mobile application development: web vs. native. Communications of the ACM, 54(5):49-53.

Chaves, J. O. M., Castro, A. F., Lima, R. W., Lima, M. V. A., and Ferreira, K. H. (2013). Mojo: Uma ferramenta de auxílio à elaboração, submissão e correção de atividades em disciplinas de programação. $33^{\circ}$ Congresso da Sociedade Brasileira de Computação (CSBC'13), $21^{\circ}$ Workshop sobre Educação em Computação (WEI' 13).

Gavalas, D. and Economou, D. (2011). Development platforms for mobile applications: Status and trends. Software, IEEE, 28(1):77-86.

Mascarenhas, D., Rubinstein, M., and Sztajnberg, A. (2008). Uma nova métrica para protocolos de roteamento em redes em malha sem fio. XXVI Simpósio Brasileiro de Redes de Computadores-SBrT.

Nurseitov, N., Paulson, M., Reynolds, R., and Izurieta, C. (2009). Comparison of json and xml data interchange formats: A case study. Caine, 2009:157-162.

Orlandi, B. H. and Isotani, S. (2012). Uma ferramenta para distribuiçao de conteúdo educacional interativo em dispositivos móveis. $23^{\circ}$ Simpósio Brasileiro de Informática na Educação.

Rabello, S., Barbosa, J. L., Oliveira, J., Wagner, A., Barbosa, D. N., and Bassani, P. B. (2012). Um modelo para colaboração em ambientes descentralizados de educação ubíqua. In Anais do $23^{\circ}$ Simpósio Brasileiro de Informática na Educação (CBIE'12), volume 23.

Santos, C. J., Franco, M. E., and Corsini, H. C. (2013). Algbr: Uma nova ferramenta para apoio ao ensino/aprendizagem de lógica computacional por meio da construção e testes de algoritmos. $33^{\circ}$ Congresso da Sociedade Brasileira de Computação (CSBC'13), $21^{\circ}$ Workshop sobre Educação em Computação (WEI' 13).

Silva, L. C. N., Neto, F. M. M., and Júnior, L. J. (2011). Mobile: Um ambiente multiagente de aprendizagem móvel para apoiar a recomendação sensível ao contexto de objetos de aprendizagem. In Anais do $22^{\circ}$ Simpósio Brasileiro de Informática na Educação (CBIE'11), $17^{\circ}$ Workshop de Informática na Escola (WIE'11), volume 1.

Vahldick, A., Mendes, A. J., Marcelino, M. J., Hogenn, M., and Schoeffel, P. (2015). Testando a diversão em um jogo sério para o aprendizado introdutório de programação. $35^{\circ}$ Congresso da Sociedade Brasileira de Computação (CSBC'15), 23 Workshop sobre Educação em Computação (WEI' 15).

Wang, G. (2011). Improving data transmission in web applications via the translation between xml and json. In Communications and Mobile Computing (CMC), 2011 Third International Conference on, pages 182-185. IEEE. 